Artículo científico

(Original paper)

\title{
REGISTRO Y DAÑO CAUSADO POR MIMOSESTES ENTEROLOBII KINGSOLVER \& JOHNSON, 1978 (COLEOPTERA: BRUCHIDAE) EN SEMILLAS DE ENTEROLOBIUM SCHOMBURGKII BENTH. (BENTHAN, 1844) (FABACEAE) EN MATO GROSSO, BRASIL
RECORD AND DAMAGE CAUSED BY MIMOSESTES ENTEROLOBII KINGSOLVER \& JOHNSON, 1978 (COLEOPTERA: BRUCHIDAE) IN SEEDS OF ENTEROLOBIUM SCHOMBURGKII BENTH. (BENTHAN, 1844) (FABACEAE) IN MATO GROSSO, BRAZIL
}

\author{
PASTOR AMADOR-MOJENA ${ }^{1 *}$, MARLITON ROCHA-BARRETO ${ }^{2}$, JESÚS ROMERO-NÁPOLES ${ }^{3}$, RODRIGO \\ CARDOSO-SNIPE ${ }^{4}$, ANTONIA A. DE SOUZA MOJENA ${ }^{5}$ \\ ${ }^{1}$ Universidad Federal de Mato Grosso, Instituto de Ciencias Agrarias y Ambientales, Av. Alexandre Ferronato, 1200. 78.557-267. \\ Sinop, Mato Grosso, Brasil. <pamadormojena@hotmail.com> \\ ${ }^{2}$ Universidad Federal de Mato Groso, Núcleo de Estudios de la Biodiversidad de La Amazonia Mato-grossense, Instituto de \\ Ciencias Naturales, Humanas y Sociales, Mato Grosso, Brasil. <mrb.ufmt@gmail.com> \\ ${ }^{3}$ Especialidad de Entomología y Acarología, Postgrado en Fitosanidad, Colegio de Postgraduados, Montecillo, Estado de México, \\ México.<jnapoles@colpos.mx> \\ ${ }^{4}$ Ingeniero Forestal, Universidad Federal de Mato Grosso. Brasil. <rodrigo.cava@ hotmail.com> \\ ${ }^{5}$ Bióloga, Rua Benedito Americo. 182. Jardín Italia 1. 78.555321. Sinop. Mato Grosso. Brasil. <aline.louro@hotmail.com> \\ *Autor corresponsal: <pamadormojena@hotmail.com>
}

Recibido: 21/10/2019; aceptado: 21/06/2020; publicado en línea: 01/07/2020

Editor responsable: Arturo Bonet Ceballos

Amador-Mojena, P., Rocha-Barreto, M., Romero-Nápoles, J., Cardoso-Snipe, R., De Souza Mojena, A. A. (2020) Registro y daño causado por Mimosestes enterolobii Kingsolver \& Johnson, 1978 (Coleoptera: Bruchidae) en semillas de Enterolobium schomburgkii Benth. (Benthan, 1844) (Fabaceae) en Mato Grosso, Brasil. Acta Zoológica Mexicana (nueva serie), 36, 1-7. https://doi.org/10.21829/azm.2020.3612247

RESUMEN. La leguminosa Enterolobium schomburgkii Benth. (Benthan, 1844) (Fabaceae), es endémica de Amazonia y posee amplia distribución geográfica en el área Neotropical. Se trata de una especie maderable de gran importancia económica. El objetivo del presente estudio fue identificar al insecto asociado a las semillas de la planta, y cuantificar y evaluar el efecto del daño provocado sobre la calidad fisiológica de las semillas recogidas en bosques urbanos en la ciudad de Sinop, Mato Grosso, Brasil. El insecto se identificó como Mimosestes enterolobii Kingsolver \& Johnson (1978). La tasa de depredación de las semillas fue de $8.07 \%$ y el consumo de sustrato fue de $0.61 \mathrm{~g}$, equivalente al $93.35 \%$ del peso de las semillas. A pesar de registrarse bajo porcentaje de daños en semillas (8.07\%), el consumo del sustrato (cotiledones y embrión de la semilla) fue alto $(93.35 \%)$, lo cual afectó significativamente la calidad fisiológica y capacidad de germinación de la semilla.

Palabras clave: Coleoptera; Bruchidae; pérdida de germinación 
Amador-Mojena, P., Rocha-Barreto, M., Romero-Nápoles, J., Cardoso-Snipe, R., De Souza Mojena, A. A. (2020) Record and damage caused by Mimosestes enterolobii Kingsolver \& Johnson, 1978 (Coleoptera: Bruchidae) in seeds of Enterolobium schomburgkii Benth. (Benthan, 1844) (Fabaceae) in Mato Grosso, Brazil. Acta Zoológica Mexicana (nueva serie), 36, 1-7. https://doi.org/10.21829/azm.2020.3612247

\begin{abstract}
The legume Enterolobium schomburgkii, Benth. (Benthan, 1844) (Fabaceae), is endemic to Amazonia and has a wide geographical distribution in the Neotropical area. It is a timber species of great economic importance. The objective of the present study was to identify the insect associated with the seeds of the plant, and quantify it, and evaluate the effect of the damage caused on the physiological quality of the seeds collected in urban forests in the city of Sinop, Mato Grosso, Brazil. The insect was identified as Mimosestes enterolobii Kingsolver \& Johnson (1978). The seed predation rate was $8.07 \%$ and the substrate consumption $0.61 \mathrm{~g}$ equivalent to $93.35 \%$ of the seed weight. Despite registering low percentage of damage to seeds $(8.07 \%$ ), the substrate consumption (cotiledons and seed embryo) was high (93.35\%), which significantly affected the physiological quality and germination capacity of the seed.
\end{abstract}

Key words: Coleoptera; Bruquidae; germination loss

\title{
INTRODUCCIÓN
}

Enterolobium schomburgkii (Benth.) Benth. es una especie endémica de Amazonia y posee una amplia distribución geográfica en el área Neotropical, desde América Central, Amazonia legal, noroeste, sudeste y sur de Brasil, extendiéndose hasta Argentina, Uruguay, Paraguay y Bolivia. Es un árbol heliófilo de 10 a 50 $\mathrm{m}$ de altura y 12 a $80 \mathrm{~cm}$ de diámetro; además de su importancia ecológica como fijadora de nitrógeno, la madera de esta especie es muy apreciada (Mesquita, 1990). Mesquita (1990) describió el fruto de $E$. schomburgkii, como indehiscente, contorcido, de color castaño a negro y de consistencia sub-leñosa, con 3-4 cm de diámetro por 1-3 cm de grosor. El epicarpio presenta superficie glabra; el mesocarpio tiene consistencia fibrosa a leñosa y una coloración castaña; el endocarpio es blanco pálido. La semilla es elíptica, de coloración amarillo azufre, con las dimensiones de $0.7 \mathrm{~cm}$ de largo por $0.4 \mathrm{~cm}$ de ancho, posee latencia física causada por impermeabilidad del tegumento al agua y es necesaria la aplicación de tratamientos de superación de latencia para lograr su germinación. Las semillas de especies forestales son depredadas por diferentes grupos de insectos; esto ocurre naturalmente en los ecosistemas, variando la intensidad del daño de un lugar a otro entre las especies y entre árboles de una misma especie; sin embargo, la acción depredadora en muchos casos llega a ser de importancia económica, debido al alto grado de semillas inviables.

Entre los insectos consumidores de semillas encontramos diversas especies de los órdenes Diptera, Lepidoptera, Coleoptera e Hymenoptera (Janzen, 1971). Dentro del orden Coleoptera, los brúquidos, conocidos comúnmente como "gorgojos", pertenecen a la familia Bruchidae y se alimentan de semillas, principalmente de leguminosas (Romero-Nápoles \& Johnson, 2004 a; b; Yus et al., 2007). El desarrollo de la etapa inmadura lo llevan a cabo dentro de las semillas, nutriéndose de los tejidos y pudiendo afectar las tasas de germinación y, por lo tanto, los procesos de mantenimiento de las especies vegetales (Ríos et al., 2005). Autores como D’Aubeterre et al. (2012), Marques et al. (2011) y Garlet et al. (2011), consideran a algunas especies de brúquidos plagas por dañar las semillas y reducir su dispersión.

En los últimos años se ha notado un interés creciente y se han desarrollado diversas investigaciones con el objetivo de conocer la diversidad, incidencia y daños de los brúquidos en diferentes partes del mundo (Luna et al., 2006; Donato et al., 2010; Garlet et al., 2011; Romero-Nápoles, 2011; Romero-Nápoles \& Westcott, 2011; Boscardin et al., 2012; de la Cruz Pérez et al., 2013; Romero-Gómez et al., 2014; LugoGarcía et al., 2015; Mojena \& Barreto, 2016). La falta de información sobre la distribución de los brúquidos 
dificulta la comprensión y pronóstico de su comportamiento, poniendo en riesgo las poblaciones vegetales susceptibles a ser atacadas y sufrir una disminución en las tasas de reproducción (Quiróz-Sodi et al., 2016). En la literatura no se encuentra registro de la asociación entre insectos y semillas de E. shomburgkii. Sólo se hace referencia, de manera aislada, a las especies de brúquidos que se han recolectado en especies de plantas de este género. El objetivo del presente estudio fue registrar el insecto asociado a las semillas de $E$. schomburgkii, determinar la ocurrencia, cuantificar y evaluar el efecto del daño provocado sobre la calidad fisiológica y capacidad de germinación de las semillas.

\section{MATERIALES Y MÉTODOS}

Frutos de varios árboles de E. shomburgkii recogidos directamente del suelo de forma aleatoria ( $11^{\circ} 51^{\text {' }}$ 33." S, 055 29' 51' O, $373 \mathrm{msnm}$ ) en el municipio de Sinop, Mato Grosso, Brasil, donde este árbol es utilizado en bosques urbanos, fueron llevados al laboratorio de semillas del Instituto de Ciencias Agrarias y Ambientales de la Universidad Federal de Mato Grosso, Campus Universitario de Sinop, para el secado de los frutos, extracción y limpieza de las semillas, realización de las pruebas de germinación, determinación de la ocurrencia y el nivel de daño.

Una muestra aleatoria de 60 frutos fue seleccionada para la extracción, secado de semillas, cálculo del número de semillas por frutos y el número de semillas por kilogramo. Para el cálculo de semillas dañadas fueron seleccionadas al azar 13 sub-muestras, en donde se contaron éstas y se clasificaron en dos categorías: sanas y dañadas. Se consideraron dañadas aquellas que presentaban señales de orificios de emergencia del imago, y/o con insectos en su interior; posteriormente se pesaron las semillas de las dos categorías en balanza electrónica de precisión de la marca Marte, modelo AY 220. Las semillas de las dos categorías fueron colocadas en recipientes independientes y mantenidas en condiciones de laboratorio (temperatura de $\left.29^{\circ} \mathrm{C} \pm 2^{\circ} \mathrm{C}\right)$ y humedad relativa del aire $(80 \% \pm 10 \%)$. Los recipientes fueron monitoreados diariamente para el conteo y el número final de insectos adultos emergidos de las semillas. Se utilizó una Lupa de Mesa Led (aumento 8X) para localizar orificios y/o la presencia del insecto en el interior de las semillas. Los insectos emergidos fueron colocados en frascos con alcohol 70\%, para su posterior identificación.

La tasa de depredación (Tp) de las semillas se calculó en base a la relación entre el número de semillas depredadas $(\mathrm{Np})$ y el total de semillas extraídas de los frutos (Ns) multiplicado X 100, utilizando la siguiente fórmula (Romero-Nápoles et al., 2005):

$$
\mathrm{Tp}=\frac{\mathrm{Np}}{\mathrm{Ns}} * 100
$$

El consumo de sustrato por los insectos fue determinado por la diferencia entre el peso de semillas sanas y depredadas, utilizando 10 muestras de 100 semillas para cada categoría, usando una balanza electrónica de precisión. Después de la cuantificación de los daños provocados por el insecto, se calculó el peso de la materia seca, el contenido de humedad de las semillas (con base en el peso fresco), así como la calidad fisiológica de éstas expresada como el porcentaje de germinación. Para determinar el contenido de humedad y el peso de la materia seca, las semillas fueron colocadas en una estufa $\left(105 \pm 3^{\circ} \mathrm{C}\right)$ por 24 horas, conforme la recomendación de las Reglas para el Análisis de Semillas (MAPA, 2009).

Para el estudio de germinación fueron seleccionadas cuatro sub-muestras de 25 semillas para cada categoría (sana y dañada), éstas fueron colocadas en cajas Gerbox con papel Germitest humedecido y colocadas en un germinador (BOD) a temperatura de $30^{\circ} \mathrm{C}$ y 12 horas luz. Para romper la latencia de las semillas sanas, se utilizó el tratamiento de escarificación mecánica lateral con lija. Las observaciones fueron diariamente, teniendo como criterio de germinación la emisión de la raíz primaria. Al finalizar la prueba, la 
cual tuvo una duración de cinco días, se determinó el porcentaje e índice de velocidad de germinación de acuerdo con las siguientes fórmulas citadas por Mojena y Barreto (2016). A las semillas no germinadas y dañadas se les realizó la prueba de viabilidad por medio de la disección de la semilla e inmersión en tetrazolio al 2\% durante 24, 48 horas. Aquellas semillas que no tenían cotiledones ni embrión se consideraron vanas.

El material está depositado en la Colección Entomológica del Instituto de Ciencias Agrarias y Ambientales de la Universidad Federal de Mato Grosso - CUS Sinop, y algunos ejemplares en la Colección de Insectos del Colegio de Postgraduados de Montecillo, Edo. de México, México (CEAM).

\section{RESULTADOS}

La especie consumiendo las semillas de E. shomburgkii fue identificada como Mimosestes enterolobii Kingsolver \& Johnson, 1978. Todavía no hay registros con relación a su distribución entre los estados brasileños; el único dato para el país es de un espécimen determinado por C. D. Johnson y depositado en la colección Texas A\&M University, Department of Entomology, USA (TAMU), con la siguiente información: Para Faz, Taperinha, 16/XI/1969, Campbell J. M. \& B.A. Campbell. Kingsolver \& Johnson (1978), describieron la especie de insectos colectados en Panamá. Posteriormente Romero-Nápoles et al. (2017) ampliaron su distribución para Costa Rica y Brasil; mencionando erróneamente como plantas hospederas a Parkinsonia aculeata L. y Prosopis juliflora (Sw.) DC., cuando en realidad, hasta el momento, la única planta hospedera para M. enterolobii es E. shomburgkii.

El daño de este brúquido se puede identificar por los orificios que dejan los adultos en la testa de la semilla al momento de la emergencia (Fig. 1A-D). Las larvas se desarrollan dentro de la semilla, permanecen durante el estado de pupa y, finalmente, los adultos emergen de las semillas de E. shumburgkii para iniciar el período reproductivo.
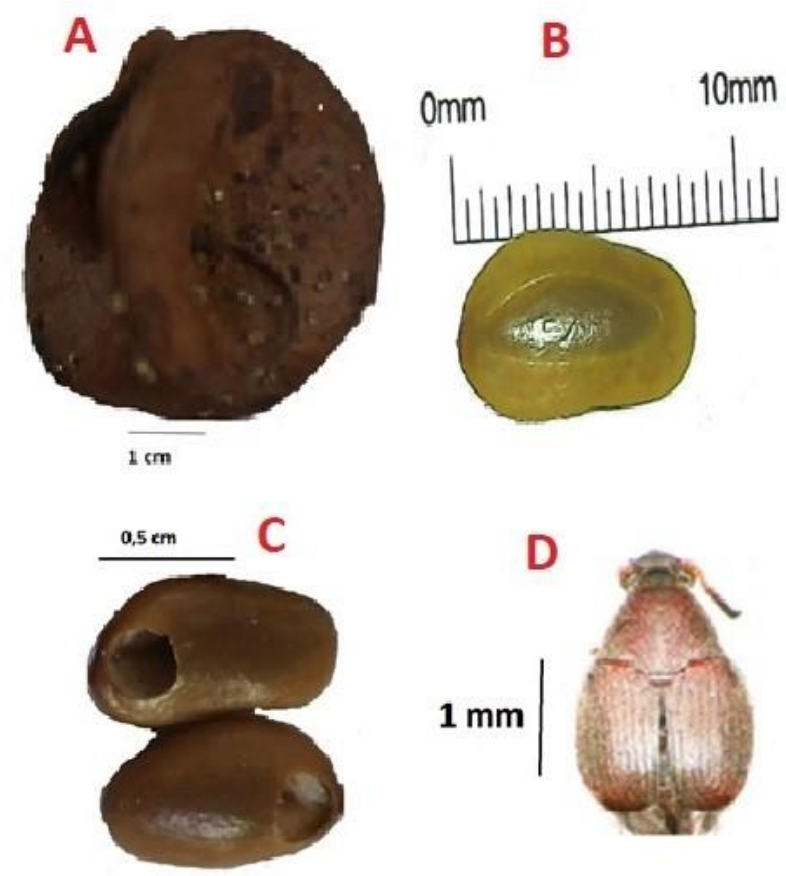

Figura 1. A) Fruto de Enterolobium shomburgkii; B) Semilla sanas; C) Semilla depredada de E. shomburgkii; D) Adulto de Mimosestes enterolobii. Escala $\mathbf{A}$ y $\mathbf{C}=\mathrm{cm}, \mathbf{B}=\mathrm{mm}$. 
El peso promedio de un fruto fresco de E. shomburgkii fue de $13.67 \mathrm{~g}$ y el número promedio de semillas por fruto fue de 23.2 ( \pm 0.25 ES); valores similares a lo reportado por Bonadeu y Santos (2013), que registraron 22-30 semillas por fruto, y por Ramos y Ferraz (2008) con 4-22. En promedio se registraron 1,522.89 semillas por kilogramo. De las 13 sub-muestras analizadas, se retiraron 1,772 semillas (136.30 semillas por sub-muestra), de las cuales 1,629 estaban sanas (91.93\%) y 143 dañadas, con una tasa de depredación (Tp) de $8.07 \%$.

El contenido de humedad de las semillas fue de 6.74\% ( $S=0.0067 ;$ Syx $=0.0030 ; C V=10.03)$. El peso promedio de una semilla sana fue de $0.66 \mathrm{~g}$, mientras que el de una semilla depredada fue de $0.04 \mathrm{~g}$. Por diferencia se obtuvo que el sustrato consumido por un insecto fue de $0.61 \mathrm{~g}$, correspondiente a una pérdida de $93.35 \%$ de la semilla.

El porcentaje de germinación de las semillas sanas fue de $100 \%$, todas germinaron en cinco días con un índice de velocidad de germinación (IVG) de 4.40. Se corroboró también que las semillas depredadas no germinan, a pesar de que éstas se mantuvieron en la cámara de germinación. En los tejidos muertos, donde no hay actividad respiratoria, las enzimas deshidrogenadas están inactivas, no ocurre reacción entre la solución de tetrazolio y, consecuentemente, las semillas permanecen descoloridas (Fig. 2 A, B).
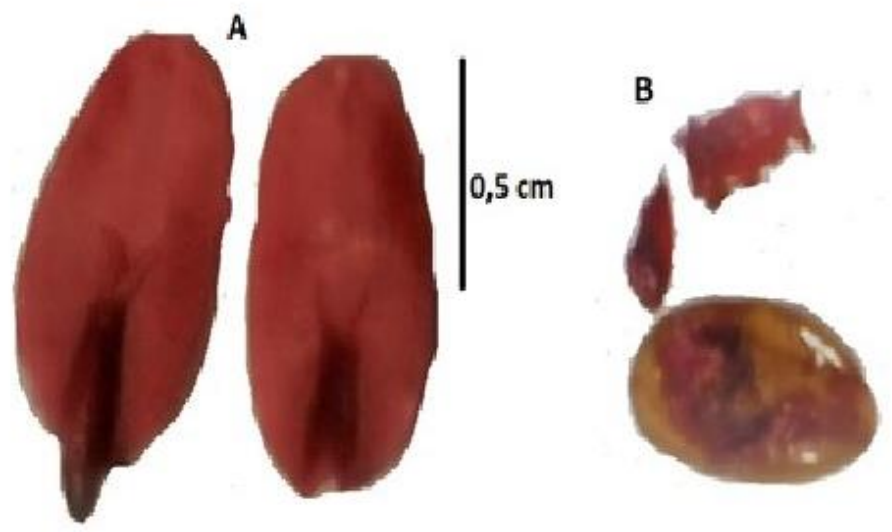

Figura 2. A) Semillas sanas mostrando el cotiledón y embrión; B) Semilla mostrando el efecto del daño causado por Mimosestes enterolobii después del teñido con la sal de tetrazolio. Escala $=\mathbf{A}$ y $\mathbf{B}$ en $\mathrm{cm}$.

\section{DISCUSIÓN}

Los brúquidos son coleópteros conocidos como "gorgojos" o "escarabajos de las semillas" y sus estados inmaduros se alimentan de semillas de alrededor de 34 familias de plantas, principalmente de leguminosas (Romero-Nápoles \& Johnson, 2004). Económicamente, este grupo destaca como una plaga primaria o secundaria al producir daños leves e incluso la pérdida total de las semillas. Aunque la mayoría de las especies de esta familia de insectos regula las poblaciones de plantas silvestres, en el futuro podrían incorporarse como plagas al ámbito productivo, por lo que el conocimiento y grado de daño de sus insectos asociados es importante para la toma de decisiones en el manejo de los productos o subproductos generados (Romero-Nápoles et al., 2005).

El género Mimosestes se encuentra exclusivamente en el Nuevo Mundo y se distribuye desde Argentina y Chile en América del Sur, al sudoeste de los Estados Unidos y el Caribe (Romero-Nápoles \& Johnson, 2004), alimentándose de una amplia diversidad de plantas hospederas (Romero-Nápoles, 2011). 
El insecto registrado en este trabajo es consumidor de semillas de E. shomburgkii, corroborando lo señalado por Ramírez y Traveset (2010), quienes citan que, entre los insectos depredadores de semillas, los de la familia Bruchidae están entre el grupo más representativo dentro del orden Coleoptera. Las larvas de este coleóptero utilizan solamente una semilla como fuente alimentaria, parece que optimizan el consumo de los tejidos de ésta, donde también encuentran cobijo.

Daños causados por insectos del género Mimosestes en semillas de especies forestales son reportados por Romero-Gómez et al. (2009) donde registran pérdidas entre 10.6 y $20.5 \%$ en Acacia pennatula. Por otro lado, Lugo-García et al. (2015), citan que las larvas de Mimosestes mimosae ocasionaron daños al 22.2\% de las semillas de Acacia cochliacanthay y 39.5\% de Parkinsonia florida. Otros brúquidos consumidores de semillas del género Enterolobiun son citados por Donato et al. (2010). Los valores registrados para $M$. enterolobii no superan a lo anotado por los autores anteriormente citados.

La prueba de tetrazolio demostró que las larvas de M. enterolobii consumieron los cotiledones y el embrión de las semillas de E. shomburgkii; sin embargo, en términos general presentaron un bajo porcentaje de semillas dañadas por las larvas.

\section{LITERATURA CITADA}

Bonadeu, F., Santos, J. U. M. dos. (2013) Contribuição ao conhecimento dos gêneros da tribo Ingeae ocorrentes em uma Floresta Nacional da Amazônia Brasileira. Rodriguésia, 64 (2), 321-336.

Boscardin, J., Redin, C. G., Costa, E. C., Longhi, S. J., Gariet, J., Watzlawick, L. F. (2012) Predação de Pseudopachymerina spinipes (Eriscson, 1833) (Coleoptera: Chrysomelidae: Bruchinae) em sementes de Vachellia caven (Molina) Seigles \& Ebinger (Fabaceae) no Parque Estadual do Espinilho em Barra do Quaraí, RS. Bioikos Campinas, 26 (2), 95-100.

D’Aubeterre, R., Principoal, J., Barrios, C., Graterol, Z. (2012) Insectos plaga en vainas de Acacia tortuosa y Prosopis juliflora para consumo animal en las zonas semiáridas del estado Lara, Venezuela. Zootecnia Tropical, 30 (2), 147-153.

de la Cruz Pérez, A., Romero-Nápoles, J., Carrillo Sánchez, J. L., García López, E., Grether González, R. G., Sanchez Soto, S., Pérez de la Cruz, M. (2013) Brúquidos (Coleoptera: Bruchidae) del estado de Tabasco, México. Acta Zoológica Mexicana (nueva serie), 29 (1), 1-95.

Donato, D. B., Fonseca, A. G., Junior, S. L. de A., Machado, E. L., Bispo, F. A. (2010) Dano de Caryedes sp. (Coleoptera; Bruchidae) e seus reflexos na propagação de Enterolobium contortisiliquum (Leguminosae). Floresta e Ambiente, 17 (2), 118-123.

Garlet, J., Costa, E. C., Boscardin, J., Murari, A. B., Machado, D. N. (2011) Damage by Stator limbatus (Horn, 1873) (Coleoptera: Chrysomelidae: Bruchinae) to Seeds of Acacia podalyriifolia A. Cunningham ex G. Don. (Fabaceae: Mimosoideae). The Coleopterists Bulletin, 65 (4), 432-433.

Janzen, D. H. (1971) Seed predation by animals. Annual Review of Ecology and Systematics, 2, 465-492.

Kingsolver, J. M., Johnson, C. D. (1978) Systematics of the genus Mimosestes (Coleoptera:Bruchidae). USDA Technical Bulletin, 1590, 1-106.

Lugo-García, G. A., López-Mora, J., Romero-Nápoles, J., Reyes-Olivas, A., Rodríguez, F., SánchezSoto, B. H. (2015) Gorgojos de la familia Bruchidae (Coleoptera) asociados a semillas de cultivos y flora adyacente del norte de Sinaloa, México. Entomología Mexicana, 2, 435-441.

Luna, L. C., Morales, N. O., Romero-Nápoles, J. (2006) Brúquidos (Coleoptera: Bruchidae) de Platanillo, Guerrero. Entomología Mexicana, 5 (2), 1054-1057.

Marques, L. H. S. F., Nakano, O., Janeiro, V., Girardi, E. A., Bueno, G. G. (2011) Biologia de Dichomeris famulata Meyrick, 1914 (Lepidoptera: Gelechiidae) em milho. Ciência Rural, 41, 108112. 
Mesquita, A. L. (1990) Revisão taxonômico de gênero de Enterolobium Mart. (LeguminosaeMimosoideae) para a região Neotropical. Dissertação de Mestrado, Universidade Federal Rural de Pernambuco, Recife, $222 \mathrm{pp}$.

MAPA (Ministério da Agricultura, Pecuária e Abastecimento) (2009) Regras para análise de sementes. Brasília: MAPA/ACS. Disponible en: http://www.agricultura.gov.br/assuntos/insumosagropecuarios/arquivos-publicacoesinsumos/2946_regras_analise_sementes.pdf

Mojena, P. A., Barreto, M. R. (2016) Daño en semillas de Enterolobium contortisiliquum (Vell) Morong por Stator harmonicus Johnson, Kingsolver y Terán, 1989 (Coleoptera: Chrysomelidae: Bruchinae) y su efecto en la germinación. Entomotropica, 31 (33), 260-266.

Quiróz-Sodi, M., Vergara-Pineda, S. S., Hernández-Sandoval, L. (2016) Nuevas especies hospederas de brúquidos (Coleoptera: Chrysomelidae) asociados a semillas de plantas nativas del estado de Querétaro, México. Entomología Mexicana, 3, 614-617.

Ramírez, N., Traveset, A. (2010) Pre-dispersal seed predation by insects in the Venezuelan Central Plain: overall patterns that influence its biology and taxonomic groups. Perspectives in Plant Ecology, Evolution and Systematics, 2 (3), 193-209.

Ramos, M. B. P., Ferraz, I. D. K. (2008) Estudos morfológicos de frutos, sementes e plântulas de Enterolobium schomburgkii Benth. (Leguminosae-Mimosoideae). Revista Brasileira de Botánica, 31 (2), 227-235.

Ríos, R. A., Romero-Nápoles, J., Carrillo, J., Bravo, H., Vera, J., Ramírez, S. (2015) Ciclo biológico y exploración de parasitismo en Specularius impressithorax (Pic) 1932 (Coleoptera: Bruchidae) en México. Acta Zoológica Mexicana (nueva serie), 31 (1), 27-35.

Romero-Nápoles, J. (2011) Gorgojos de las semillas (Insecta: Coleoptera: Bruchidae). Pp: 361-365. En: A. Cruz Aragón (Ed.). La biodiversidad en Veracruz: Estudio de Estado. Comisión Nacional para el Conocimiento y Uso de la Biodiversidad (CONABIO). Gobierno del Estado de Veracruz, Universidad Veracruzana, Instituto de Ecología, A.C., México.

Romero-Gómez, G., Romero-Nápoles, J., Ramos, R. Y., Solorio, A. B., Carrasco, J. V., Morales, A. F. (2009) Gorgojos de la familia Bruchidae (Coleoptera) asociados a semillas de plantas silvestres destinadas para germoplasma. Boletín Sociedad Entomológica Aragonesa, 44, 333-342.

Romero-Gómez, G., Romero-Nápoles, J., Solorio, A. B., Sánchez, J. L. C., Mojica, H. B., Alarcón, S. R. (2014) Brúquidos (Coleoptera: Bruchidae) del estado de Morelos, México. Acta Zoológica Mexicana (nueva serie), 30 (1), 1-17.

Romero-Nápoles, J., Johnson, C. D. (2004a) Sinopsis de los brúquidos de México (Insecta: Coleoptera). Pp: 3. 758-763. En: M. Morales, A. M. G. Ibarra, A. del P. G. Rivera, S. C. Stanford (Eds.). Entomología Mexicana. Sociedad Mexicana de Entomología.

Romero-Nápoles, J., Johnson, C. D. (2004b) Checklist of the Bruchidae (Coleoptera) of Mexico. The Coleopterists Bulletin, 58 (4), 613-635.

Romero-Nápoles, J., Westcott, R. L. (2011) The Bruchidae (Insecta: Coleoptera) of la Reserva de la Biósfera Sierra de Huautla, Morelos, Mexico, with descriptions of two new species and an annotated checklist. Insecta Mundi, 0166, 1-15.

Romero-Nápoles, J., Grether, G. R., Camargo, S. L. R., Johnson, C. D. (2005) Método para la evaluación de daño de semillas por brúquidos (Insecta: Coleoptera) en el campo, con nuevos registros de hospederos y distribución para el grupo. Pp: 4. 107-111. En: M. A. Morales, M. G. Ibarra, A. E. Mendoza, M. P. G. Ibarra, S. C. Stanford (Eds.). Entomología Mexicana. Colegio de Postgraduados-SME. Montecillo, Texcoco, Edo. de México.

Romero-Nápoles, J., Kingsolver, J. M., Yus, R. R. (2017) Checklist of the Bruchidae (Coleoptera: Chrysomeloidea) of Costa Rica. Boletín de la Sociedad Entomológica Aragonesa (S.E.A.), 60, 153 166.

Yus, R. R., Kingsolver, J. M., Romero, N. J. (2007) Sobre el estatus taxonómico actual de los brúquidos (Coleoptera: Bruchidae) en los Chrysomeloidea. Dugesiana, 14 (1), 1-21. 